\title{
Caracterização física e química de substratos com diferentes proporções de resíduo de celulose ${ }^{(1)}$
}

\author{
MAXIMILIANO KAWAHATA PAGLIARINI(2), REGINA MARIA MONTEIRO DE CASTILHO(3), \\ ERICA RODRIGUES MOREIRA ${ }^{(4)}$, MARLENE CRISTINA ALVES ${ }^{(5)}$
}

\begin{abstract}
RESUMO
O objetivo do trabalho foi caracterizar física e quimicamente substratos contendo diferentes proporções de resíduo de celulose. Os componentes de substrato utilizados foram: solo, areia e resíduo de celulose, compondo os tratamentos: T1 = Resíduo de celulose $(\mathrm{RC}) ; \mathrm{T} 2=\mathrm{RC}+\operatorname{solo}(1: 1) ; \mathrm{T} 3=\mathrm{RC}+\operatorname{solo}(1: 2) ; \mathrm{T} 4=\mathrm{RC}+\operatorname{solo}(1: 3) ; \mathrm{T} 5=\mathrm{RC}+\operatorname{areia}(1: 1) ; \mathrm{T} 6=\mathrm{RC}+\operatorname{areia}(1: 2) ;$ $\mathrm{T} 7=\mathrm{RC}+$ areia $(1: 3) ; \mathrm{T} 8=\mathrm{RC}+$ solo + areia $(1: 1: 1) ; \mathrm{T} 9=\mathrm{RC}+$ solo + areia $(1: 2: 1) ; \mathrm{T} 10=\mathrm{RC}+$ solo + areia $(1: 3: 1) ; \mathrm{T} 11$ $=\mathrm{RC}+$ solo + areia $(1: 1: 2) ; \mathrm{T} 12=\mathrm{RC}+$ solo + areia $(1: 1: 3)$. Avaliou-se a densidade do substrato, capacidade de recipiente, $\mathrm{pH}$ e condutividade elétrica. $\mathrm{O}$ resíduo de celulose puro se adequou em relação às propriedades físicas de um substrato para produção de mudas. Em relação à condutividade elétrica o substrato mais adequado para esse fim foi obtido misturando-se resíduo de celulose com solo e areia. $\mathrm{O}$ pH mostrou-se alto em todos os substratos.
\end{abstract}

Palavra-chave: Densidade, $\mathrm{pH}$, condutividade elétrica.

\begin{abstract}
Physical and chemical characterization of substrates with different proportions of cellulose residue

The objective of this study was to characterize physic and chemically substrates with different proportions of cellulose residue. The components of substrate used were soil, sand and cellulose residue and the treatments were: T1 = cellulose residue (CR); $\mathrm{T} 2=\mathrm{CR}+$ soil $(1: 1) ; \mathrm{T} 3=\mathrm{CR}+$ soil $(1: 2) ; \mathrm{T} 4=\mathrm{CR}+$ soil $(1: 3) ; \mathrm{T} 5=\mathrm{CR}+$ sand $(1: 1) ; \mathrm{T} 6=\mathrm{CR}+$ sand $(1: 2) ; \mathrm{T} 7=\mathrm{CR}+$ sand (1:3); T8 $=\mathrm{CR}+$ soil + sand $(1: 1: 1) ; \mathrm{T} 9=\mathrm{CR}+$ soil + sand $(1: 2: 1) ; \mathrm{T} 10=\mathrm{CR}+$ soil + sand $(1: 3: 1) ; \mathrm{T} 11=\mathrm{CR}+$ soil + sand (1:1:2); T12 $=\mathrm{CR}+$ soil + sand (1:1:3). It was evaluated the substrate density, container capacity, $\mathrm{pH}$ and electrical conductivity. The pure cellulose residue adapts in respect of physical properties. In relation to electrical conductivity the best results were obtained by mixing cellulose residue with soil and sand. The $\mathrm{pH}$ determined in water was high in all substrates. Keywords: Density, $\mathrm{pH}$, electrical conductivity.
\end{abstract}

\section{INTRODUÇÃO}

Substrato é definido como o meio onde se desenvolvem as raízes das plantas cultivadas na ausência de solo (KÄMPF, 2005), que deve servir para fixá-las, suprir suas necessidades de ar, água e nutrientes (LEMAIRE, 1995; TAVEIRA, 1996; SALVADOR, 2000).

Os substratos têm sua utilização mundial por proporcionarem melhores condições físicas, químicas e biológicas ao desenvolvimento das plantas (KÄMPF, 2005). Esses materiais são formados por diferentes matérias-primas e classificados de acordo com o material de origem (ABREU, 2002): origem vegetal (xaxim, esfagno, turfa, carvão, fibra de coco e resíduos de beneficiamento como tortas, bagaços e cascas); origem mineral (vermiculita, perlita, granito, calcário, areia e cinasita) e origem sintética (lã de rocha, espuma fenólica e isopor) (FERRAZ et al., 2005).
Além desses materiais há a geração de resíduos no setor florestal, nesse caso as fábricas de papel e celulose deparamse com problemas de ordem ambiental, devido à grande quantidade de resíduos gerados (BELLOTE et al., 1998).Os mesmos autores afirmam ainda que a aplicação dos resíduos orgânicos, oriundos da fabricação de celulose e papel pode trazer benefícios como incorporação de nutrientes minerais necessários às árvores florestais; melhoria das propriedades físicas como granulometria, capacidade de retenção de água e densidade do solo. Além disso, a aplicação de resíduos de celulose e cinza de caldeiras tem efeito positivo na atividade biológica do solo acelerando a decomposição da serapilheira e no aumento da ciclagem de nutrientes.

Costa etal. (2002) observaram em pesquisa realizada com resíduo de celulose que promoveu aumentos significativos no $\mathrm{pH}$ do substrato favorecendo o desenvolvimento das plantas, principalmente pelo aumento da disponibilidade

\footnotetext{
(1) Trabalho recebido para publicação em 22/05/2012 e aprovado em 26/02/2015

(2) Universidade Estadual Paulista "Júlio de Mesquita Filho", Departamento de Fitotecnia, Tecnologia de Alimentos e Sócio-Economia, Ilha SolteiraSP. *Autor correspondente: max.pagliarini@gmail.com.

(3) Universidade Estadual Paulista "Júlio de Mesquita Filho", Departamento de Fitotecnia, Tecnologia de Alimentos e Sócio-Economia, Avenida Brasil, Centro, 56, CEP 15385-000, Ilha Solteira-SP.

(4) Universidade Estadual Paulista "Júlio de Mesquita Filho", Departamento de Fitossanidade, Engenharia Rural e Solos, Avenida Brasil, Centro, 56, CEP: 15385-000, Ilha Solteira-SP.
} 
dos principais nutrientes minerais, redução do $\mathrm{Al}^{+3}, \mathrm{Mn}^{+2}$ livres e de $\mathrm{H}^{+}$na solução do solo. Apesar da liberação de

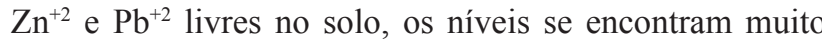
abaixo dos valores máximos permitidos pelas legislações nacional e internacional sem comprometimento do solo e da qualidade dos produtos agrícolas produzidos.

Porém, nesses materiais sozinhos dificilmente se encontram todas as características necessárias para atender às condições para o ótimo crescimento e desenvolvimento das plantas (SOUZA, 1995). Nesse sentido, o substrato poderá ser formado por parte de solo mineral e parte de matéria orgânica, de um ou de diversos materiais ou misturas.

O substrato considerado ideal para a produção de mudas, seja de espécies florestais bem como de espécies ornamentais, é aquele que apresenta uniformidade em sua composição, sendo isento de pragas, microorganismos fitopatogênicos e sementes de plantas daninhas. Essas características eliminam a necessidade de se proceder à sua desinfestação, concorrendo para diminuir os custos de produção das plantas (CAMPINHOS JÚNIOR e IKEMORI, 1983). Além disso, algumas características físicas e químicas devem ser observadas como densidade, capacidade de recipiente, condutividade elétrica e $\mathrm{pH}$.

Em relação à densidade, materiais menos densos podem acarretar problemas na fixação das plantas e tombamento do recipiente, se o cultivo é feito em recipientes altos. No entanto, quando o cultivo é feito em bandejas, necessitase de substratos leves, pois as baixas densidades não comprometem a estabilidade do recipiente. Além disto, as baixas densidades permitem a utilização desses materiais como condicionadores em misturas com outros materiais de alta densidade como o solo (SANTOS, 2006).

A capacidade de recipiente, que mede a capacidade de retenção de água, é um termo introduzido por White e Mastalerz (1966), que se refere ao volume de água retido no substrato após saturação e livre drenagem, sem sofrer evaporação. Ou, ainda, segundo Martínez et al. (1991), a capacidade de recipiente é a máxima capacidade de retenção de água de um substrato em um determinado recipiente, sob as mesmas condições de saturação e drenagem.

Santos et al. (2002) observaram que substratos alternativos como bagaço de cana, casca de arroz e serragem apresentaram características físicas apropriadas para a produção de mudas; também Schmitz et al. (2002) observaram essas características em substrato formado por turfa vermelha + resíduo decomposto de casca de acácia negra. Em contrapartida, Guerrine e Trigueiro (2004) não obtiveram características físicas apropriadas à produção de mudas em substratos compostos por casca de arroz e biossólidos.

Em relação às propriedades químicas, a condutividade elétrica (CE) é um indicativo da concentração de sais ionizados na solução (WILSON, 1984) e fornece um parâmetro estimado da salinidade do substrato sendo que, para cada espécie, essa salinidade pode afetar algum processo metabólico. Há espécies que são mais sensíveis durante o processo de germinação e, após este período, vão se ajustando paulatinamente ao estresse salino. Há aquelas que toleram maior nível de salinidade até a emergência das plântulas e são menos tolerantes durante a fase de crescimento. Há também as que são mais fortemente afetadas durante a floração e frutificação que por ocasião do processo germinativo e crescimento inicial (BERNSTEIN, 1964; AYERS e WESTCOT, 1991).

Com relação ao $\mathrm{pH}$ os substratos devem apresentar valores dentro de uma faixa considerada adequada para o cultivo de plantas, pois os valores inadequados, além de influenciar a disponibilidade de nutrientes (CARNEIRO, 1995), estão relacionados a desequilíbrios fisiológicos na planta (WILSON, 1983).

Ribeiro et al. (2005) observaram que mudas de maracujá-amarelo produzidas em substrato comercial composto de casca de pinus, em sacos plásticos, obtiveram melhor resposta para todas as características físicas e químicas avaliadas em relação ao substrato. Carrijo et al. (2004) sugerem que a fibra de coco verde pode constituir um excelente substrato para o cultivo de tomate em ambiente protegido possibilitando se obter alta produtividade com qualidade. Lopes et al. (2008) observaram que as características químicas do substrato formado por casca de pinus, vermiculita e casca de coco foram ideais para utilização como substrato. Já para Zietemann e Roberto (2007), o substrato ideal para formação de mudas de goiabeira 'Paluma' e 'Século XXI' são a mistura de solo + areia + matéria orgânica (esterco de curral) $(2: 1: 1)$ e o substrato comercial Plantmax ${ }^{\circledR}$.

$\mathrm{O}$ objetivo do trabalho foi caracterizar física $\mathrm{e}$ quimicamente substratos contendo diferentes proporções de resíduo de celulose.

\section{MATERIAL E MÉTODOS}

O experimento foi conduzido nos Laboratórios de Horticultura da Faculdade de Engenharia da UNESP - Campus de Ilha Solteira - SP, onde foram realizadas as análises físicas e químicas dos substratos contendo diferentes proporções de resíduo de celulose.

$\mathrm{O}$ delineamento experimental foi inteiramente casualizado, com doze tratamentos e quatro repetições. Os materiais utilizados para a formulação dos substratos foram: solo, areia e resíduo de celulose, compondo os seguintes tratamentos: $\mathrm{T} 1=$ Resíduo de celulose $(\mathrm{RC}) ; \mathrm{T} 2$ $=\mathrm{RC}+$ solo $(1: 1) ; \mathrm{T} 3=\mathrm{RC}+$ solo $(1: 2) ; \mathrm{T} 4=\mathrm{RC}+$ solo $(1: 3) ; \mathrm{T} 5=\mathrm{RC}+$ areia $(1: 1) ; \mathrm{T} 6=\mathrm{RC}+$ areia $(1: 2) ; \mathrm{T} 7=$ $\mathrm{RC}+\operatorname{areia}(1: 3) ; \mathrm{T} 8=\mathrm{RC}+$ solo + areia $(1: 1: 1) ; \mathrm{T} 9=\mathrm{RC}+$ solo + areia $(1: 2: 1) ; \mathrm{T} 10=\mathrm{RC}+$ solo + areia $(1: 3: 1) ; \mathrm{T} 11=$ $\mathrm{RC}+$ solo + areia $(1: 1: 2) ; \mathrm{T} 12=\mathrm{RC}+$ solo + areia $(1: 1: 3)$.

O solo utilizado foi Latossolo Vermelho Distroférrico (Empresa Brasileira de Pesquisa Agropecuária - Embrapa, 2006) retirado da camada de $0-20 \mathrm{~cm}$ da Fazenda Experimental da UNESP, Campus de Ilha Solteira-SP localizada no município de Selvíria-MS; a areia média foi lavada e o resíduo de celulose formado por resto de casca de eucalipto de uma indústria de papel e celulose do município de Três Lagoas-MS. Na Tabela 1 são apresentados os resultados das análises químicas do resíduo de celulose, realizado pelo Centro de P\&D de Solos e Recursos Ambientais do Instituto Agronômico de Campinas-SP. 
Tabela 1. Análises químicas do resíduo de celulose realizadas pelo Centro de P\&D de Solos e Recursos Ambientais do Instituto Agronômico de Campinas-SP, UNESP, Ilha Solteira - SP, 2010.

Table 1. Chemical analysis of the residue of pulp made by the $R \& D$ Center of Soil and Environmental Resources of the Agronomic Institute of Campinas-SP, UNESP, Ilha Solteira - SP, 2010.

\begin{tabular}{|c|c|c|c|c|c|c|c|c|c|c|c|}
\hline \multirow[t]{2}{*}{ Componente } & Umidade & C orgânico & $\mathrm{N}$ & $\mathrm{Ca}$ & S & $\mathrm{P}$ & $\mathrm{Mg}$ & B & $\mathrm{Cu}$ & $\mathrm{Fe}$ & $\mathrm{K}$ \\
\hline & ----\%"----- & \multicolumn{6}{|c|}{ 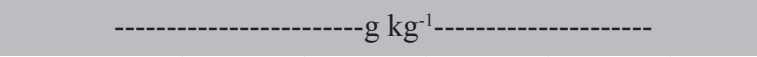 } & \multicolumn{4}{|c|}{----------'mg kg-1------- } \\
\hline R. de celulose & 5,9 & 186 & 6,3 & 86,9 & 1,8 & 2,4 & 3,8 & 30,3 & 14,3 & 5,45 & 5.95 \\
\hline
\end{tabular}

Método de ensaio: para metais - US-EPA, SW-846, método 3051, com determinação por fotômetro de chama para Na e K, para os demais metais determinação por ICP-AES. Para N total método de Kjeldahl, para carbono orgânico digestão com dicromato e determinação volumétrica, para umidade perda de massa a $60^{\circ} \mathrm{C}$.

Realizaram-se análises físicas (densidade e capacidade de recipiente) dos substratos conforme metodologia citado por Kämpf et al. (2006). A condutividade elétrica e o pH foram determinados respectivamente pelo condutivímetro TDSTestr 4 e pHmetro pHTestr 2, ambos da Oakton Instruments $^{\circledR}$, colocando-se cada substrato em repouso por quatro horas em água destilada na proporção de 1:1,5 (v:v, substrato e água), segundo metodologia adaptada de Kämpf (2005), sendo a leitura feita na solução sobrenadante.

Os resultados foram submetidos à análise de variância com auxilio do programa Sisvar (FERREIRA, 2011) e, quando significativos, as médias foram comparadas pelo Teste de Scott-Knott, ao nível de 5\% de probabilidade.

\section{RESULTADOS E DISCUSSÃO}

Em relação à densidade do substrato, observa-se na Tabela 2 que os tratamentos T7, T11 e T12 apresentaram as maiores médias $\left(998,61 ; 1.009,72\right.$ e $1.029,86 \mathrm{~kg} \mathrm{~m}^{-3}$, respectivamente) diferindo estatisticamente dos demais tratamentos. Em contrapartida, T1 apresentou a menor densidade, $526,39 \mathrm{~kg} \mathrm{~m}^{-3}$.

Tabela 2. Densidade do substrato (Ds) e capacidade de recipiente (CRec) dos substratos com diferentes proporções de resíduo de celulose $(\mathrm{RC}=$ resíduo de celulose; $\mathrm{S}=$ solo; $\mathrm{A}=$ areia $)$. Ilha Solteira, $\mathrm{SP}, 2012$.

Table 2. Substrate density and container capacity of substrate with different proportion of cellulose residue (RC $=$ cellulose residue; $S=$ soil; $A$ = sand. Ilha Solteira, SP, 2012.

\begin{tabular}{|c|c|c|c|c|}
\hline \multirow{3}{*}{$\begin{array}{l}\text { Substratos } \\
\text { (T1) RC }\end{array}$} & \multicolumn{2}{|c|}{ Ds } & \multicolumn{2}{|c|}{ CRec } \\
\hline & \multicolumn{2}{|c|}{------ kg m³----- } & \multicolumn{2}{|c|}{------ \% ------- } \\
\hline & 526,39 & $\mathrm{f}$ & 48,01 & $\mathrm{a}$ \\
\hline (T2) $\mathrm{RC}+\mathrm{S}(1: 1)$ & 725,00 & e & 32,18 & b \\
\hline (T3) $\mathrm{RC}+\mathrm{S}(1: 2)$ & 782,64 & d & 29,21 & $\mathrm{c}$ \\
\hline (T4) $\mathrm{RC}+\mathrm{S}(1: 3)$ & 811,11 & d & 26,79 & d \\
\hline$(\mathrm{T} 5) \mathrm{RC}+\mathrm{A}(1: 1)$ & 887,50 & $\mathrm{c}$ & 23,52 & $\mathrm{c}$ \\
\hline (T6) $\mathrm{RC}+\mathrm{A}(1: 2)$ & 970,14 & $\mathrm{~b}$ & 16,24 & g \\
\hline (T7) $\mathrm{RC}+\mathrm{A}(1: 3)$ & 998,61 & a & 13,58 & $\mathrm{~h}$ \\
\hline (T8) $\mathrm{RC}+\mathrm{S}+\mathrm{A}(1: 1: 1)$ & 888,20 & c & 24,68 & $\mathrm{c}$ \\
\hline (T9) $\mathrm{RC}+\mathrm{S}+\mathrm{A}(1: 2: 1)$ & 919,45 & $\mathrm{c}$ & 21,92 & $\mathrm{f}$ \\
\hline$(\mathrm{T} 10) \mathrm{RC}+\mathrm{S}+\mathrm{A}(1: 3: 1)$ & 896,53 & $\mathrm{c}$ & 21,47 & f \\
\hline$(\mathrm{T} 11) \mathrm{RC}+\mathrm{S}+\mathrm{A}(1: 1: 2)$ & $1.009,72$ & $\mathrm{a}$ & 16,92 & g \\
\hline$(\mathrm{T} 12) \mathrm{RC}+\mathrm{S}+\mathrm{A}(1: 1: 3)$ & $1.029,86$ & a & 15,31 & g \\
\hline DMS & 12,01 & & 0,71 & \\
\hline F & 142,095 & & 178,52 & \\
\hline CV (\%) & 2,76 & & 5,86 & \\
\hline
\end{tabular}

Médias seguidas por letras distintas na coluna diferem estatisticamente entre si pelo teste de Scott-Knott a 5\% de probabilidade. 
De maneira geral, considera-se como referência para substrato valores de densidade entre 350 e $600 \mathrm{~kg} \mathrm{~m}^{-3}$ (BUNT, 1973), sendo que apenas o resíduo de celulose se encontra dentro deste intervalo (Tabela 2).

Porém, o substrato indicado para recipientes de 15 cm de altura não necessariamente apresenta uma boa performance em recipientes menores do que cinco centímetros (MILKS et al., 1989), ou seja, quanto menor o recipiente menor deve ser a densidade do substrato utilizado. Kämpf (2005) recomenda utilizar substratos com densidade de 100 a $350 \mathrm{~kg} \mathrm{~m}^{-3}$ para bandejas multicelulares, de 250 a $400 \mathrm{~kg} \mathrm{~m}^{-3}$ para vasos de até 15 $\mathrm{cm}$ de altura, de 300 a $550 \mathrm{~kg} \mathrm{~m}^{-3}$ para vasos de 20 a 30 $\mathrm{cm}$ de altura e, de 500 a $800 \mathrm{~kg} \mathrm{~m}^{-3}$ para vasos maiores de $30 \mathrm{~cm}$ de altura. Neste trabalho, nenhum substrato é recomendado para bandejas multicelulares e vasos de até
$15 \mathrm{~cm}$ de altura, já em relação aos vasos de 20 a $30 \mathrm{~cm}$ de altura recomenda-se apenas o substrato $\mathrm{T} 1$ e para vasos maiores que $30 \mathrm{~cm}$, recomendam-se os substratos T2 e T3 (Tabela 2).

Com relação à capacidade de recipiente (Tabela 2), o tratamento 1 apresentou maior média (48,01\%) diferenciando estatisticamente dos demais tratamentos. Já os substratos T6, T11 e T12 obtiveram as menores médias de capacidade de recipiente $(16,24 \% ; 16,92 \%$ e $15,31 \%$ respectivamente). Fermino (2003) recomenda para um substrato capacidade de recipiente entre 20 e 30\% de retenção de água, nesse caso os melhores substratos foram T3, T4, T5, T8, T9 е T10.

Observa-se na Tabela 3 que o substrato T1 diferenciouse estatisticamente dos demais tratamentos apresentando $\mathrm{pH}$ igual a 8,60 .

Tabela 3. Condutividade elétrica (CE) e pH das combinações de componentes de substrato com resíduo de celulose (RC= resíduo de celulose; $\mathrm{S}=$ solo; $\mathrm{A}$ = areia). Ilha Solteira, SP, 2012.

Table 3. Electric conductivity and pH of combination of substrate components with cellulose residue (RC $=$ cellulose residue; $S=$ soil; $A=$ sand). Ilha Solteira, SP, 2012.

\begin{tabular}{|c|c|c|c|c|}
\hline \multirow{3}{*}{$\begin{array}{l}\text { Substratos } \\
\text { (T1) RC }\end{array}$} & \multirow{2}{*}{\multicolumn{2}{|c|}{$\begin{array}{c}\mathrm{pH} \\
\mathrm{em} \mathrm{H}_{2} \mathrm{O}\end{array}$}} & \multirow{2}{*}{\multicolumn{2}{|c|}{$\begin{array}{c}\mathrm{CE} \\
\mathrm{dS} \mathrm{m}^{-1}\end{array}$}} \\
\hline & & & & \\
\hline & 8,60 & $\mathrm{a}$ & 1,63 & $\mathrm{a}$ \\
\hline (T2) RC + S (1:1) & 8,28 & $\mathrm{~b}$ & 0,83 & $\mathrm{c}$ \\
\hline (T3) $\mathrm{RC}+\mathrm{S}(1: 2)$ & 8,10 & $\mathrm{~b}$ & 0,88 & $\mathrm{c}$ \\
\hline (T4) $\mathrm{RC}+\mathrm{S}(1: 3)$ & 8,13 & $\mathrm{~b}$ & 0,70 & d \\
\hline (T5) $\mathrm{RC}+\mathrm{A}(1: 1)$ & 8,05 & $\mathrm{~b}$ & 1,00 & $\mathrm{~b}$ \\
\hline (T6) $\mathrm{RC}+\mathrm{A}(1: 2)$ & 8,13 & $\mathrm{~b}$ & 0,65 & d \\
\hline (T7) $\mathrm{RC}+\mathrm{A}(1: 3)$ & 8,18 & $\mathrm{~b}$ & 0,58 & $\mathrm{e}$ \\
\hline (T8) $\mathrm{RC}+\mathrm{S}+\mathrm{A}(1: 1: 1)$ & 8,05 & $\mathrm{~b}$ & 0,70 & d \\
\hline (T9) $\mathrm{RC}+\mathrm{S}+\mathrm{A}(1: 2: 1)$ & 8,05 & $\mathrm{~b}$ & 0,58 & $\mathrm{e}$ \\
\hline$(\mathrm{T} 10) \mathrm{RC}+\mathrm{S}+\mathrm{A}(1: 3: 1)$ & 8,15 & $\mathrm{~b}$ & 0,48 & $\mathrm{e}$ \\
\hline$(\mathrm{T} 11) \mathrm{RC}+\mathrm{S}+\mathrm{A}(1: 1: 2)$ & 8,13 & $\mathrm{~b}$ & 0,48 & $\mathrm{e}$ \\
\hline$(\mathrm{T} 12) \mathrm{RC}+\mathrm{S}+\mathrm{A}(1: 1: 3)$ & 8,15 & $\mathrm{~b}$ & 0,45 & $\mathrm{e}$ \\
\hline DMS & 0,06 & & 0,05 & \\
\hline F & 5,07 & & 40,99 & \\
\hline CV (\%) & 1,64 & & 13,68 & \\
\hline
\end{tabular}

Médias seguidas por letras distintas na coluna diferem estatisticamente entre si pelo teste de Scott-Knott a 5\% de probabilidade.

Segundo Fermino (2003), valores de pH em água não levam em conta apenas a acidez total, especialmente devido aos prótons e às formas de alumínio fixadas ao complexo de troca, que contribuem também para a acidez. Assim, os valores encontrados no presente trabalho são elevados, provavelmente por terem sido lidos em água. Pagliarini et al. (2010) também observaram elevados valores de $\mathrm{pH}$ em água misturando-se resíduo de celulose e componentes de substratos, corroborando com o presente trabalho. Costa et al. (2002) observaram em pesquisa realizada com resíduo de celulose que este promoveu aumentos significativos no pH ao misturá-lo com o substrato.
$\mathrm{O}$ pH considerado ideal, para cultivo de plantas em solo está em torno de 6,0, conforme recomendação da COMISSÃO DE QUÍMICA E FERTILIDADE DO SOLO - RS e SC (2004). Porém, quando se usa substratos orgânicos, sem solo, recomenda que o pH deve estar na faixa de 5,2 a 5,5 (K ̈̈MPF, 2005). Os valores de pH encontrados no resíduo de celulose e em misturas com solo e areia, se assemelham aos citados por Kämpf (2005) para vermiculita (entre 7,5 - 8,5) e para compostos de lixo urbano (entre 8,0 - 8,6).

Segundo Martinez (2004) o efeito indireto do $\mathrm{pH}$ diz respeito à solubilidade dos nutrientes, sendo que em $\mathrm{pH}$ 
superior a 6,0 pode ocorrer precipitação dos mesmos deixando de ser disponível para as plantas, principalmente o nitrogênio, que está intimamente ligado à formação das moléculas de clorofila. Cadahía e Eymar (1992) afirmaram que o intervalo ótimo de $\mathrm{pH}$ para o bom crescimento vegetativo em substrato está entre 5,0 - 6,5, bem inferior ao $\mathrm{pH}$ apresentado por todos os tratamentos.

Observa-se na Tabela 3 que a condutividade elétrica (CE) no substrato T1 obteve o maior valor $(1,63 \mathrm{dS}$ $\mathrm{m}^{-1}$ ), enquanto que T7, T9, T10, T11 e T12 apresentaram as menores $\mathrm{CE}\left(0,58 ; 0,58 ; 0,48 ; 0,48\right.$ e $0,45 \mathrm{dS} \mathrm{m}^{-1}$, respectivamente).

No presente trabalho, apenas os substratos T2, T3 e T5 se encontram na faixa sugerida por Cavins et al. (2000) que afirmam que um substrato com $\mathrm{CE}$ entre 0,76 a $1,25 \mathrm{dS}$ $\mathrm{m}^{-1}$ apresenta salinidade adequada ao desenvolvimento da maioria dos cultivos. Os mesmos autores classificam $\mathrm{CE}$ em níveis: muito baixo $\left(0\right.$ a $\left.0,25 \mathrm{dS} \mathrm{m}^{-1}\right)$, baixo $(0,26$ a 0,75 $\left.\mathrm{dS} \mathrm{m} \mathrm{m}^{-1}\right)$, normal $\left(0,76\right.$ a $\left.1,25 \mathrm{dS} \mathrm{m}^{-1}\right)$, alto $(1,26$ a $1,75 \mathrm{dS}$ $\mathrm{m}^{-1}$ ), muito alto $\left(1,76\right.$ a $2,25 \mathrm{dS} \mathrm{m}^{-1}$ ) e extremo (acima de 2,25 $\mathrm{dS} \mathrm{m}^{-1}$ ). Assim sendo os substratos T4, T6, T7, T8, T9, T10, T11 e T12 se enquadram em um nível baixo de CE; os substratos T2, T3 e T5 nível normal e o substrato T1 se enquadra em nível alto.

$\mathrm{O}$ resíduo de celulose puro se adequou em relação às propriedades físicas de um substrato para produção de mudas.

\section{CONCLUSÃO}

Com base nos resultados obtidos e nas condições deste experimento pode-se concluir que o resíduo de celulose puro de acordo com suas características físicas (densidade do substrato e capacidade de recipiente) pode ser utilizado como substrato na produção de mudas.

Em relação à condutividade elétrica os melhores resultados foram obtidos misturando-se resíduo de celulose com solo e areia, em qualquer proporção.

$\mathrm{O}$ pH determinado em água mostrou-se alto em todos os substratos.

\section{REFERÊNCIAS}

ABREU, M.F. Uso da análise química na avaliação da qualidade de substratos e componentes. In: ENCONTRO NACIONAL DE SUBSTRATOS PARA PLANTAS, 3., 2002, Campinas. Anais... Campinas: IAC, p.17-28, 2002.

AYERS, R.S.; WESTCOT, D.N. A qualidade da água na agricultura. Campina Grande: UFPB, 1991. 218p.

BELlOTE, A.F.J.; FERREIRA, C.A.; SILVA, H.D. da; ANDRADE, G. de C. Resíduos da indústria de celulose em plantios florestais. Boletim de Pesquisa Florestal, Colombo, v.6, n.16, p. 99-106, 1998.

BERNSTEIN, L. Salt tolerance of plants. Washington: [s.n.], 1964. 23p. (Information Bulletin, 283).
BUNT, A.C. Some physical and chemical characteristics of loamless pot-plant substrate and this relation to plant growth. Plant and Soil, The Hogue, v.38, p.1954-1954, 1973.

CADAHÍA, C.; EYMAR, E. Caracterización química y fisioquímica sustrato. Actas de Horticultura, Córdoba, v.11, n. 3, p.19-25, 1992

CAMPINHOS JÚNIOR, E.; IKEMORI, Y.K. Novas técnicas para produção de mudas de essências florestais. IPEF, Piracicaba, n.23, p.47-52, 1983.

CARNEIRO, J.G.A. Produção e controle de qualidade de mudas florestais. Curitiba: UFPR/FUPEP, 1995. 451p.

CARRIJO, O.A.; VIDAL, M.C.; REIS, N.V.B.; SOUZA, R.B.; MAKISHIMA, N. Produtividade do tomateiro em diferentes substratos e modelos de casas de vegetação. Horticultura Brasileira, Brasília, v.22, n. 1, p.05-09, 2004.

CAVINS, T.J.; WHIPKER B.E.; FONTENO, W.C.; HARDEN, B.; McCALL, I.; GIBSON, J.L. Monitoring and managing $\mathrm{pH}$ and $\mathrm{EC}$ using the Pour Thru Extraction Method. Horticulture Information Leaflet / NCSU, Raleigh, n. 590, 2000. Disponível em: $<$ http://www2.ncsu. edu/unity/lockers/project/hortsublab/>. Acesso em: 01de setembro de 2010.

COMISSÃO DE QUÍMICA E FERTILIDADE DO SOLO - RS E SC. Manual de adubação e de calagem para os estados do Rio Grande do Sul e Santa Catarina. 10ed. Porto Alegre, 2004, 400p.

COSTA, A.S.V. da; GALVÃO, E.R.; LOVO, I.C.; FERRARI JUNIOR, M.J.; ALMEIDA, L.L.; BENEVIDES, G. Efeitos do resíduo de celulose nas características químicas dos solos e no desenvolvimento de culturas agrícolas. In: CONGRESSO E EXPOSIÇÃO ANUAL DE CELULOSE E PAPEL, 35, 2002, São Paulo. Anais... São Paulo: Associação Brasileira Técnica de Celulose e Papel, p.01-04, 2002.

FERMINO, M.H. Métodos de análises para caracterização física de substratos para plantas. 2003. 104f. Tese (Doutorado), Pós-Graduação em Fitotecnia, UFRGS, Porto Alegre, 2003.

FERRAZ, M.V.; CENTURION, J.F.; BEUTLER, A.N. Caracterização física e química de alguns substratos comerciais. Acta Scientiarum Agronomy, Maringá, v.27, n.2, p.209-214, 2005.

FERREIRA, D.F. Sisvar: a computer statistical analysis system. Ciência e Agrotecnologia. Lavras, v.35, n.6, p.1039-1042, 2011.

GUERRINE, I.A.; TRIGUEIRO, R.M. Atributos físicos e químicos de substratos compostos por biossólidos e casca de arroz carbonizada. Revista Brasileira de Ciência do Solo, Viçosa, v.28, p.1069-1076, 2004. 
KÄMPF, A.N. Produção comercial de plantas ornamentais. Guaíba: Agrolivros, 2005, 256p.

LEMAIRE, F. Physical, chemical and biological properties of growing medium. Acta Horticulturae, Wageningen, v.396, p.273-284, 1995.

LOPES, J.L.W.; GUERRIN, I.A.; SAAD, J.C.C.; SILVA, M.R. da. Atributos químicos e físicos de dois substratos para produção de mudas de eucalipto. Cerne, Lavras, v.14, n.4, p.358-367, 2008.

MARTINEZ, H.E.P. Distúrbios nutricionais em hortaliças cultivadas em substratos com baixa atividade química. In: BARBOSA, J.G.; MARTINES, H.E.P.; PEDROSA, M.W.; SEDIYAMA, M.A.N. (Eds.). Nutrição e Adubação de Plantas Cultivadas em Substratos. Viçosa: UFV, p.129157, 2004.

MARTÍNEZ, P.F.; BURÉS, S.; BLANCA, F.; YUSTE, M.P.; VALERO, J. Experimental and theorical air/water ratios of different substrate mixtures at container capacity. Acta Horticulturae, Wageningen, v.294, n.4, p.241-248, 1991.

MILKS, R.R.; FONTENO, W.C.; CARSON, R.A. Hidrology of agricultural substrate: III. Predicting air and water content of limited-volume plug cells. Journal of the American Society for Horticulturae Science, Alexandria, v.114, n.1, p.57-61, 1989.

PAGLIARINI, M.K.; CASTILHO, R.M.M.; ALVES, M.C. Caracterização física de misturas de componentes de substrato com resíduo de celulose para fins de produção de mudas. In: ENCONTRO NACIONAL DE SUBSTRATO PARA PLANTAS, 7. 2010, Goiânia. Anais... Goiânia: UFG, 2010.

RIBEIRO, M.C.C.; MORAIS, M.J.A. de; SOUZA, A.H. de; LINHARES, P.C.F.; BARROS JÚNIOR, A.P. Produção de mudas de maracujá-amarelo com diferentes substratos e recipientes. Caatinga, Mossoró, v.18, n.3, p.155-158, 2005.

SALVADOR, E.D. Caracterização física e formulação de substratos para o cultivo de algumas ornamentais. 2000. Tese (Doutorado em Agronomia, Produção Vegetal)
- Escola Superior de Agricultura Luiz de Queiroz, Universidade de São Paulo, Piracicaba, 2000.

SANTOS, F.G.B. dos. Substratos para produção de mudas utilizando resíduos agroindustriais. 2006. 79f. Dissertação (Mestrado em Ciência do Solo) - Faculdade de Agronomia, Universidade Federal Rural de Pernambuco, Recife, 2006.

SANTOS, F.R.P.; CASTILHO, R.M.M. de; DUARTE. E.F. Caracterização físico-química de sete componentes de substratos recomendados para uso em floricultura. Cultura Agronômica, Ilha Solteira, v.11, n.1, p.81-92, 2002.

SOUZA, M.M. Avaliação de substratos para o cultivo de crisântemo (Chrysanthemum morifolium Ramat., compositae) 'White Polaris' em vasos. Revista Brasileira de Horticultura Ornamental, Campinas, v.1, n.2, p.71$77,1995$.

SCHMITZ, J.A.K.; SOUZA, P.V.D. de; KÄMPF, A.N. Propriedades químicas e físicas de substratos de origem mineral e orgânica para o cultivo de mudas em recipientes. Ciência Rural, Santa Maria, v.32, n.6, p.937-944, 2002.

TAVEIRA, J.A. Substratos - cuidados na escolha do tipo mais adequado. 1996, 2p. (Boletim Ibraflor Informativo, 13).

WHITE, J.W.; MASTALERZ, J.W. Soil moisture as related to "Container Capacity". Procedings of the American Society for Horticulturae Science, Geneva, v.89, n.2, p.758-765, 1966.

WILSON, G.C.S. Analytical analyses and physical properties of horticultural substrates. Acta Horticulturae, Wageningen, v.150, n.6, p.19-32, 1984.

WILSON, G.C.S. Tomato production in bark substrate. Acta Horticulturae, Wageningen, v.150, n.7, p.271-276, 1983.

ZIETEMANN, C.; ROBERTO, S.R. Produção de mudas de goiabeira (Psidium guajava L.) em diferentes substratos. Revista Brasileira de Fruticultura, Jaboticabal, v.29, n.1, p.137-142, 2007. 